(b) it gave every man the opportunity to develop his own personality, and neither religion nor ethnic origin was any bar [Prof. Saha has overlooked the fact that though this is so constitutionally, yet in practice there is the Negro problem]; (c) the people had the genius to develop the resources of the country, using the latest discoveries in science and technology ; (d) there has been a great administrative centre for the whole country, which did not allow any constituent State to secede, or interfere with internal development. Should not we in India remember these great lessons on the eve of our impending political changes ?"

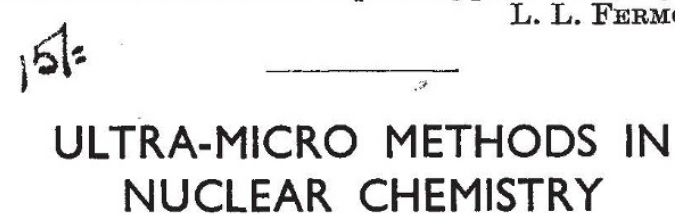

$\mathrm{G}$ T. SEABORG haq agtein added to the general knowledge of thrirans-uranic elements by his introductory r $\phi \mathrm{m}$ of s to a meeting of the American Chemical Socibty and his lecture there on "The Impet Nuclear Chemistry"'. According to the rat tentative definition with which Seaborg prefaces his introductory address, "nuclear chemistry should be defined as embracing the chemical aspects of the study and application of nuclear reactions, the investigation of radio-chemistry, and the application of radio-active isotopes and nuclear methods to the study of chemical problems in general".

The interest of the paper centres in Seaborg's description of the ultra-microchemical methods used in the investigation of the trans-uranic elements. Immediately after the discovery of the first isotopes of neptunium and plutonium, namely, $\mathrm{Np} 239$ (May, 1940), $\mathrm{Np} 238$ and $\mathrm{Pu} 238$ (late in 1940), $\mathrm{Pu} 239$ (spring, 1941) and $\mathrm{Np} 237$ (early in 1942), their chemical properties could be studied only by tracer methods on carriers provided by elements of lower atomic number. However, as it was intended to work out at that early stage the process to be used for the large-scale extraction of plutonium once the piles were working, it was most desirable to test the methods in advance with visible and weighable amounts of material. Therefore, microgram quantities of plutonium and neptunium were produced by prolonged irradiation of uranium with cyclotrons; in order to get concentrations comparable to those in the technical application, the reactions were performed in minute amounts of solution $\left(10^{-1}\right.$ $10^{-5}$ c.c.). These are handled with the help of capillary containers, pipettes and burettes. Liquid volumes are measured in calibrated capillary tubing, the movement of the liquid within the capillary being governed by air pressure under sensitive control. The smaller pipettes may be built to fill automatically by capillary attraction. The test-tubes and beakers for this work are made out of capillary tubing with an inside diameter of $0 \cdot 1-1 \mathrm{~mm}$. The weights of solids handled amount to about $0 \cdot 1-100$ micrograms; a precision of 0.5 per cent is easily reached. Levels are observed under the microscope. Pipettes, etc., are worked with micro-manipulators. Liquids are usually separated from solids by centrifugation rather than filtration.

For weighings, the Salvioni balance is often employed. This has a pan supported by a horizontal quartz fibre, which bends when weights are applied. As an alternative, a balance was developed in the
University of California with which $1 \mu \mathrm{gm}$. or less, in a container of up to $25 \mathrm{mgm}$., can be weighed with an accuracy of 2 per cent. In this balance, a beam, to which the two pans are fixed, is supported at right angles to the plane of the beam by a horizontal quartz fibre. When the beam is depressed on one side under a load, it is restored to the initial horizontal position by twisting the supporting quartz fibre. The twist needed is a measure of the load.

Up to 1943 , altogether about $1,000 \mu \mathrm{gm}$. plutonium (239) were produced in cyclotrons. Not only were reactions in the liquid phase investigated, but also dry reactions, and even the properties of the metal itself. Smaller, but still weighable, quantities of neptunium were also made. In this case, the isotope $\mathrm{Np} 237$ was used, being the only known isotope of neptunium of long half-life $\left(2.25 \times 10^{6}\right.$ years $)$. This is the daughter of the $\beta$-active $U 237$ formed by the $\mathrm{U} 238(n, 2 n) \mathrm{U} 237$ reaction. As the latter process is highly endothermic and, therefore, needs fast neutrons, yields are much smaller. Nowadays, of course, $\mathrm{Np} 237$ as well as $\mathrm{Pu} 239$ are made in the piles in quantities sufficient for the application of ordinary chemical methods ${ }^{2}$.

Seaborg's researches on elements 95 and 96 have been referred to before ${ }^{2}$. He has now named the two elements 'americium' and 'curium'. The name "americium', given to the element with six $5 f$ electrons, is in analogy to the name 'europium' of the element with six $4 f$ electrons. The name 'curium' for the element with seven $5 f$ electrons, recalling the pioneers of radioactivity, corresponds to the name 'gadolinium', given to the element with seven $4 f$ electrons in honour of the pioneer of the investigation of the rare earths.

It is expected by Seaborg that the starting of the new 184-in. cyclotron in Berkeley will give a new impetus to nuclear chemistry. Employing particles of up to $400 \mathrm{MeV}$. energy, far-reaching destruction even of lighter nuclei with the emission of showers of protons and neutrons as well as new types of fission are anticipated, leading to new families of radioactive isotopes.

E. BRODA

1 Chem. and Eng. Neus (May 10, 1946), 1192.

Nature, 157, 307 (1946).

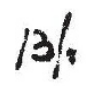

\section{IDENTIFICATION OF THE CORONAL LINES}

DROF. BENGT EFIEN delivered the George 1 Darwin Dorte of the Royal Astronomical spolect, The Identification of the Coronal Lines". As (a) result of all observations of the coronal liff spectrum, the wave-lengths of about twenty coronal emission lines have been established. Six outstanding lines are due to iron, namely, $\lambda \lambda 3388$, $5303,6374,7892,10747$ and 10798. No coronal lines have been observed in a laboratory source of light, and coronal lines were a unique feature of the solar corona until 1932, when a number of them were observed in the spectrum of the recurrent nova $R S$ Ophiuchi. Coronal lines reappeared in Nova Ophiuchi at the 1942 outburst and were also observed in 1945 in $T$ Pyxidis.

The evidence regarding the nature of 'coronium' is carefully examined, and as a result the conclusion 
is reached that the essential part of the coronal lines is due to iron, nickel, calcium and argon atoms deprived of about half their normal electron envelope. Such a high state of ionization raises certain problems in solar phenomena, and various attempts have been made to give a physical explanation to established facts.

The existence of a high temperature in the corona is supported by a number of arguments, among which may be noticed the following, all of which point to a temperature exceeding a quarter of a million degrees : (1) the mean high state of ionization as revealed by the emission lines ; (2) the breadth of the emission lines, if this is due to thermal Doppler effect, though macroscopic irregular motions or radial motions of matter might cause the broadening of the lines ; (3) the blurring out of the Fraunhofer lines in the continuous spectrum of the inner corona, assumed to be an effect of the velocities of the scattering electrons; (4) the absence of the Balmer lines in the emission line spectrum of the corona, explained by the electrons being too fast to be captured by the protons; (5) dynamical considerations showing that great thermal velocities are necessary to balance the gravitational forces in order to explain the observed density gradient of the corona.

Reference is made to the theory of Alfven that the corona might consist of particles with very high energy; Alfvén derived from the density function a temperature of about a million degrees. A quotation is given from Alfvén's work which explains the heating mechanism: "Motion of solar matter in magnetic fields on the sun, especially the vortical motion in a sunspot, must bring about a potential difference between different points of the solar surface, and it was shown that under certain conditions this gives rise to discharges above the surface of the sun. Calculations indicate that the electromotive force can be as high as $10^{7}$ volts, so that even if charged particles are usually accelerated only by a small fraction of this potential, they attain rather high energies. The process is most conspicuous in the prominences, where consequently we can expect a very intense production of high energy particles. As the mechanism is of a very general character the same process is likely to take place very frequently on a smaller scale. If-as many authors mean-we can regard the chromosphere as a multitude of small prominences, it is likely that a production of high energy particles takes place almost anywhere on the solar surface or in some layer above it."

Menzel held a different view of the origin of the highly ionized particles in the corona, and suggested that the coronal matter was ejected from the hot interior of the sun through holes and cracks on the surface. More recently, Vegard has expressed a somewhat similar opinion; he thinks that the highly ionized heavy ions in the corona come from the sun's deeper layers, being driven away from the sun at high speeds by means of the electric fields resulting from the photo-electric effect produced by soft $\mathrm{X}$-rays. Saha has suggested that the highly ionized atoms emitting the coronal lines are the fragments of a kind of nuclear fission occurring near the surface of the sun.

At present it would be unwise to pronounce in favour of any one of these views, and the physical explanation of the corona still remains a problem.
BROADCASTING IN GREAT BRITAIN

THE British Broadfofthy Corporation is at present operating enter the terms of a Royal Charter which campinto force on January 1, 1937, for a period of th years (see Nature, 139, 19; 1937). In view $f e^{\circ}$ approaching expiration of this charter, the Goxpmiment has given careful consideration to the Arability of appointing an independent body to advise on the organisation of British broadcasting in the future. But it has been decided that such an inquiry would not be appropriate at the present time ; and that the charter and licence granted to the B.B.C. should be renewed, with certain alterations, for a period of five years from January 1, 1947.

The justification for this action is given in a White Paper* recently published. This paper forms a concise historical review of broadcasting in Great Britain, outlining the policy adopted throughout and the development and activities of the Home and Overseas Servicas up to and including the war years. On the matter of the appropriateness of an inquiry, it is stated that though the Government is not opposed in principle to the appointment of an independent committee, there are three main reasons for not doing this at the present time or even in one year's time. In the first place, since September 1939, the B.B.C. have been operating under completely abnormal conditions; and the existing charter and licence have therefore run for less than three years under normal conditions; this is an insufficient period to enable a sound judgment to be made of the merits or otherwise of the organisation established in 1937. Secondly, it is too early yet to see with any clarity the effects on peace-time broadcasting of the remarkable developments in the field of electronics and radiofrequency technique which have taken place during the past ten years. Thirdly, the broadcasting service in Britain must operate with due regard to the allocation of wave-lengths for all radio purposes by the International Telecommunications Union; and it will inevitably be some time before the existing agreements can be revised to take account of the geographical and technical changes of the past six years.

In renewing the charter and licence for a period of five years, the Government proposes to consider well in advance of the expiry of this period the desirability of appointing an independent committee to advise on future broadcasting. In the meantime, the B.B.C. will continue to provide its service as a public utility body which is ultimately responsible to Parliament, but which is free so far as possible to carry out its obligations without political interference or control. The Postmaster-General is responsible to Parliament for the broadcasting vote, while the Lord President of the Couneil now deals with all questions of major broadcasting policy.

The White Paper gives details of the distribution before and during the War of the wave-lengths available to Great Britain, and describes the present arrangement of national and regional stations, and the corresponding programmes. A third programme for Home listeners, intended to be received effectively by about 80 per cent of the population, will be introduced by the B.B.C. in the autumn. An obliga. tion has been laid on the Corporation to broadcast a

* Broadcasting Policy. (Cmd. 6852.) Pp. 28. (London : H.M. Stationery Office, 1946.) 6d. net. 